Widdowson, E. M. \& McCance, R. A. (1936). F. Hyg., Camb., 36, 293.

Wolff, H. S. (1956). Proc. Nutr. Soc. 15, 77.

Woolf, B. (1954). Proc. Nutr. Soc. 13, 82.

Yudkin, J. (195I). Brit. F. Nutr. 5, 177.

\title{
Genetic variation in the nutrition of Drosophila melanogaster-some general inferences
}

By Forbes W. Robertson, Agricultural Research Council Unit of Animal Genetics, Institute of Animal Genetics, Edinburgh

\section{Introduction}

To consider genetic variation in the nutritional requirements of Drosophila melanogaster at a symposium where most of the interest centres on man would seem to call for some justification. The physiology and development of fly and mammal might appear sufficiently different to limit the usefulness of comparisons between them. With respect to quantitative requirements for specific nutrients this may be quite true, but is less relevant when we consider the more general problem of genetic variation in relation to diet. By virtue of the Mendelian basis of genetic variation, data culled from any population of diploids add to the common store of concepts and models which is drawn upon whenever we try to interpret variation between individuals of any species, including man. Variation within and between populations is discussed in terms of breeding structure, population size, inbreeding, fluctuations in selection pressure, mutation, gene flow between populations and the significance of deviations from the mean, for survival and reproduction, in different traits.

During the last 15 years or so experimental work with Drosophila, the mouse, the hen and other species has established an organized approach to polygenic variation and to methods for describing its properties. Because of differences in life cycle, some species are better suited than others for tackling certain problems and, of course, in Drosophila the genetic analysis can be taken furthest. The genetic behaviour of similar traits in these widely differing species has much in common, judged by the effects of selection and inbreeding. This is especially true of characters such as body size, growth rate and survival that play corresponding roles in the general economy. There is therefore sound reason for looking at evidence for genetic variation in the nutritional requirements of Drosophila for clues to what we might, or should, look for in man.

I shall describe some of the results of experiments I have carried out during the last 2 or 3 years, some already published (Robertson, I $960 a, b, c$; Prabhu \& Robertson, 1961 ), others in preparation, illustrating only the general features. These experiments were not designed to study genetic variation in nutritional requirements in quite the same way as is commonly understood in the field of nutrition. They arose as part of a general study of the properties of genetic variation, which influences body

21 (2) 5 
size and growth rate. Earlier genetic analysis had been carried out with unrestricted diets, and in due course the analysis called for study of how far the phenotypic effects of genetic differences in size and growth rate could be modified by altering the diet in different ways. Deficiencies in protein and also ribonucleic acid (RNA), which the animal cannot synthesize fast enough for maximum growth rate, are the commonest limiting factors in the usual environment, and so most of the data relate to comparisons of performance on diets in which one or other of these nutrients was present in suboptimal concentrations.

The general nutritional requirements of Drosophila are well known. The animal can be grown aseptically on defined media in which protein, in the form of casein, RNA, cholesterol, lecithin, fructose, seven B-vitamins and also salts are incorporated in an agar gel. Thus, with constant temperature, we can effect quite a rigorous control of the environment. The criteria of performance here refer to the size of the adults and the duration of the larval period. Various tests have suggested that the larva has to reach a critical size early in the third instar before the next stage of growth, preceding pupal differentiation, can be entered on. Prolongation of development time apparently belongs to the first stage, since, after the critical point has been passed, development time is comparatively unaffected by variation in diet, although the growth in mass may be greatly altered. Thus the relations between change in body size and development time allow us to make inferences about whether the first or the second stage of growth or both are involved. Such a distinction acquires further interest in so far as the passage from one stage to the next is almost certainly controlled by shifts in hormonal balance which are related to the onset of differentiation.

With respect to the numerical values recorded in the accompanying tables and figures, body size refers to the length of the thorax, a more reliable measure of insect size than weight. The records have been transformed to $3 \times \ln$ thorax length so that multiplying the differences between means by 100 gives values roughly equivalent to percentage differences in weight, which helps to keep their relative magnitude in perspective. Larval period is expressed as $\ln$ days. The animals used in these experiments have all been derived from the same foundation population.

A convenient way of detecting gene-environment interaction is to select for size or speed of growth on a particular diet and then compare the performance of selected strains and unselected controls on this and other diets. In the experiments described here the comparisons have been carried out after only a few generations of selection, and the number of parents in successive generations has been relatively large so that inbreeding may be discounted as a significant factor in the origin of the observed differences.

\section{Experimental results}

Variance on suboptimal diets. Table I shows the within-culture variance of unselected individuals, when they were grown on the favourable live-yeast medium and also on defined diets in which either fructose was absent or the level of RNA or protein was reduced. There was highly significant heterogeneity of variance 
and, on the low-protein diet, the variance was twice as great as on the live-yeast medium. Even the omission of fructose, which involves the comparatively small reduction of some $10 \%$ in size, clearly increased the variance.

Table I. Within-culture variance of body size of Drosophila melanogaster, measured as three times In thorax length (in $\left.\mathrm{mm} \times \mathrm{1}^{-2}\right)$, on different diets

\begin{tabular}{lccc}
\multicolumn{1}{c}{ Diet } & $\begin{array}{c}\text { Mean } \\
\text { body size }\end{array}$ & $\begin{array}{c}\text { Mean square } \\
\times \text { I }^{2}\end{array}$ & df \\
Live yeast & 14.08 & 0.39 & I32 \\
No fructose & 13.98 & 0.52 & I I \\
Low-ribonucleic acid & 13.85 & 0.55 & I I I \\
Low-protein & 13.75 & 0.77 & IO8
\end{tabular}

Selection on low-protein diets. From the physical homogeneity of the environment it might be inferred that this increase in variability is genetic, i.e. the effects of segregating genes is enhanced on these suboptimal diets. To pursue this further, selection for large size was carried out on a low-protein diet, on a medium in which all nutrients were reduced to one-third the usual concentration, and also on the unrestricted live-yeast medium. Many tests and comparisons have been carried out, but I can deal here with only some of the more suggestive results.

Selection on the low-protein diet led to a substantial increase in size, some $25 \%$ compared to unselected controls grown on this medium. But this difference was diminished in comparisons made with the live-yeast medium (Fig. I). Thus the

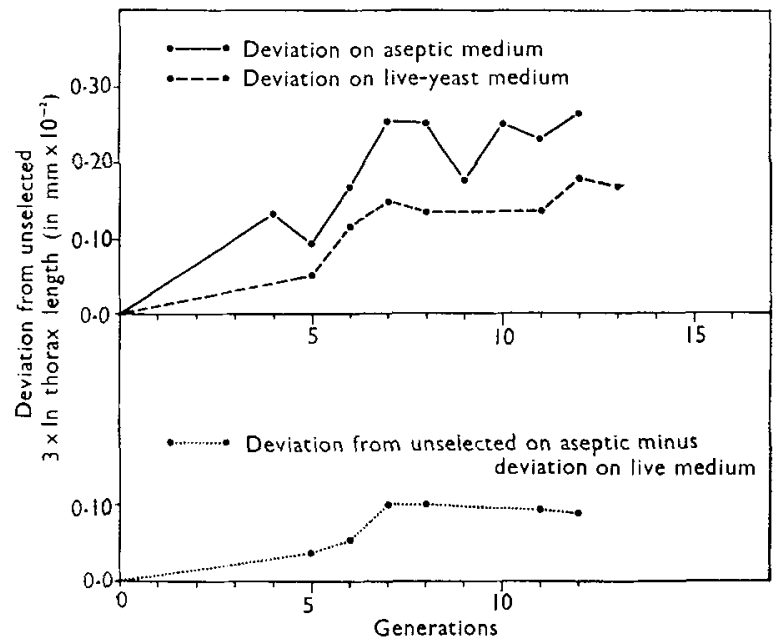

Fig. I. Selection of Drosophila melanogaster for large body size on low-protein diet.

increased variance on the low-protein diet included genetic effects which were manifested under these particular conditions, but not on the yeast medium, and selection quickly established gene arrays which conferred ability to grow to larger size on this particular diet. Along with this increase in performance the within-culture variance declined below the level of the unselected controls, as is shown in Fig. 2, 
which also includes similar information for the large line selected on the diluted medium. The jump at generation 5 was probably due to infection and is of no special significance.

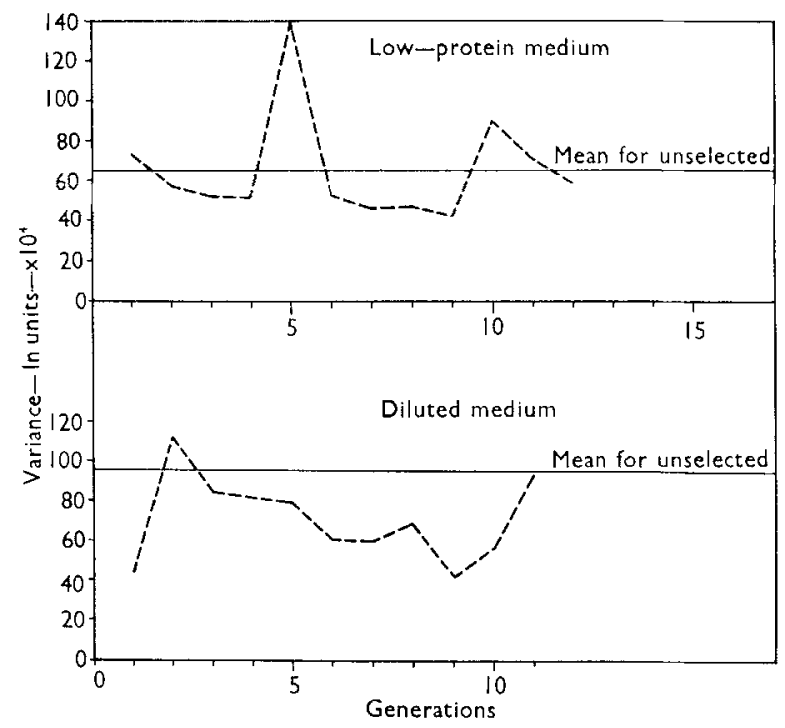

Fig. 2. Within-culture variance of body size in selected strains of Drosophila melanogaster.

Further evidence of genetic differentiation according to the composition of the diet was provided by growing the unselected controls and the three different large lines on various suboptimal media. Thus, with different levels of protein the lines selected on the low-protein and the diluted media showed little reduction in size until the protein concentration was reduced about $40 \%$ below the standard level. The unselected strain, and also the large line selected on the yeast medium, suffered a much greater decline. With further reduction in protein content the difference between the controls and the lines selected on suboptimal media became greater, and at the lowest protein level the line selected on the live-yeast medium was very drastically reduced in size. Thus the three large lines which are about the same size on the yeast medium differ strikingly in their reaction to low-protein diet in a way obviously related to the nutritional conditions during their selection.

Selection on low-RNA media. We have now to consider what happens when the diet is made suboptimal in a different way, namely by cutting down the level of RNA but keeping the protein content high. In addition to selecting for larger size in four lines, selection for shorter development time was also carried out on the low-RNA medium. It may be noted that on the favourable live-yeast medium selection for shorter development time is generally quite ineffective, which is hardly surprising since shorter development time makes an important contribution to fitness under competitive conditions. 
The results of these experiments differ clearly from those of experiments carried out with the low-protein diets in that selection for larger size was regularly accompanied by roughly proportional increase in the duration of the larval period, whereas, in the lines considered earlier, development time did not exceed that of the unselected controls. This striking contrast is shown in Fig. 3. Also, selection for faster development time was accompanied by a correlated reduction in body size. Thus the diets

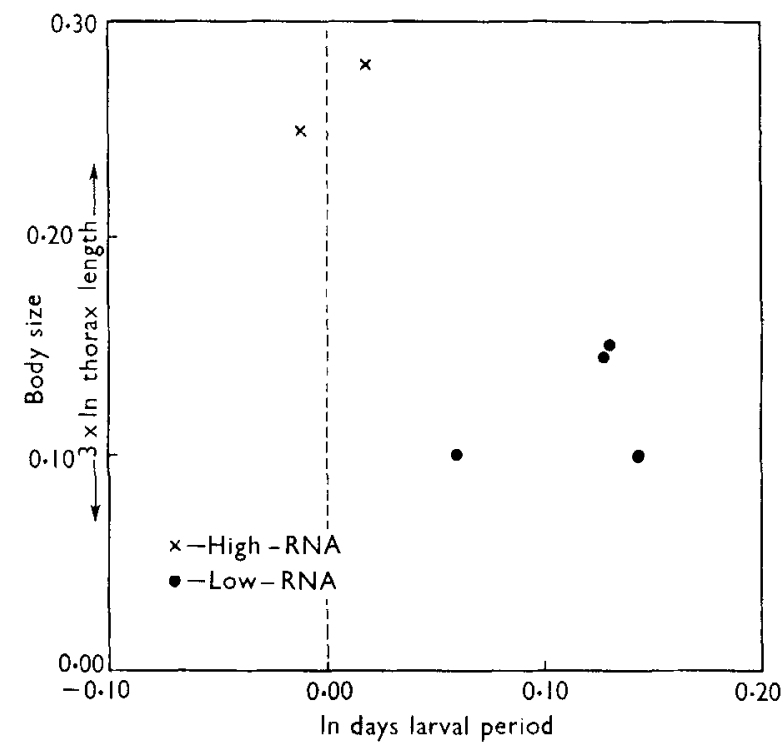

Fig. 3. Selection of Drosophila melanogaster for large size on diets which differ in RNA : protein ratiodeviation from unselected.

which differed in RNA : protein ratio have exposed different genetic effects which influence development in qualitatively different ways-perhaps acting specifically in either the earlier or the later stages of larval growth. Thus, statistically similar increases in individual variation on different suboptimal diets are qualitatively different with respect to developmental origin.

When the lines selected on the low-RNA diet are transferred to the unrestricted live-yeast medium, the large lines retain their characteristically larger size and take longer to develop, but the lines selected for shorter development time, though remaining smaller in size, now either do not differ from the unselected controls, or take longer to develop. Many different tests have shown that the realization of their potentially shorter development time is restricted to a narrow range of conditions which are being studied further. Such behaviour is consistent, of course, with all the other evidence suggesting that natural selection has established gene arrays which hold development time to a minimal level in the environment to which the population is adapted.

In another test, selection was carried out for shorter and longer development time on a diet which lacked fructose, but in which all other nutrients were kept at a high level. This medium is probably equivalent to a mildly protein-deficient diet. In 
sharp contrast with the results of selecting on the low-RNA diet, selection for shorter development time was entirely without effect, and body size also remained unchanged. Selection in the other direction, for longer larval period, led to a clear-cut increase in this character but no change in body size. Thus the kind of physiological change is a function of the composition of the larval diet during apparently similar selection. We have here a fruitful approach to interrelationships between diet, genotype and physiology.

Effects of inbreeding. The last piece of evidence from Drosophila relates to differences in reaction to nutritional variation on the part of inbred lines and crosses between them, and raises the general question of what is to be regarded as suitable test material in laboratory animals. A number of inbred lines have been established by close inbreeding from the same population which gave rise to the selected lines we have already considered. These inbred lines and the various crosses between them have been grown on a variety of suboptimal diets. Considering first the comparisons between the non-inbred population and the inbred lines on media lacking fructose or low in RNA or low in protein or with all nutrients diluted to one-third of standard concentration, we find striking differences in how far body size is reduced below the level on the unrestricted live-yeast medium. The heterogeneity for each treatment shown in Table 2 is statistically highly significant and some of the differences are quite dramatic. It may be noted that inbred lines do not necessarily suffer

Table 2. Reaction of inbred lines and original population of Drosophila melanogaster to different suboptimal diets measured as deviations from body size on unrestricted yeast diet (ln scale)

$\begin{array}{ccccc}\text { Inbred line no. } & \text { No fructose } & \begin{array}{c}\text { Low ribonucleic } \\ \text { acid }\end{array} & \text { Diluted } & \text { Low protein } \\ 3 & -0.01 & 0.00 & -0.17 & -0.29 \\ 4 & -0.07 & -0.21 & -0.33 & -0.27 \\ 6 & -0.04 & -0.16 & -0.30 & -0.20 \\ 7 & -0.07 & -0.32 & -0.27 & -0.49 \\ \text { I0 } & -0.01 & -0.27 & -0.28 & -0.28 \\ \text { Mean for inbreds } & -0.05 & -0.19 & -0.27 & -0.31 \\ \text { Population } & -0.15 & -0.31 & -0.29 & -0.27\end{array}$

greater decline than the non-inbred controls and with the diets lacking fructose, and with diets low in RNA, the inbred individuals, on average, suffer less decline than the non-inbred. Thus each inbred line comprises a unique gene array with its own unpredictable pattern of response to different kinds of nutritional variation. When the inbred lines are crossed, we find that the performance of the $F_{1}$ closely resembles the average performance of the highly heterozygous foundation population in both size and development time on the live-yeast medium and also on different suboptimal diets. A statistical test of gene-environment interaction reveals a high level of heterogeneity for the inbred lines, but a much lower value for the crosses and, when one atypical cross is excluded, the heterogeneity is no longer statistically significant.

These observations are relevant to the generality of inferences about nutritional requirements for any laboratory animal, especially the familiar rat. A good deal of 
nutritional work has been carried out with inbred lines or with populations which, although they may not have been deliberately or consistently inbred, nevertheless approximate to inbred lines by virtue of their origin from a few individuals and frequent restriction of population size. Although inbred lines have the advantage of being genetically homogeneous, it is rash to suggest that what is true of one or two lines is also true of rats in general. Strictly speaking, extrapolation from rat to man would be most reliable in studies on wild rats. Since this is not practicable, crosses between unrelated inbred lines might provide more reliable and representative material for research.

\section{General inferences}

Turning now to the general implications of these experiments, we must recognize that individual variation in any response to diet on the part of man or other species will be partly genetic in origin, and that restricted diets will tend to increase the level of variability due to segregation. This effect will contribute to differences between results reported by different investigators, especially when the sample size is small. It will be true of individuals drawn from the same population and, in comparisons between distinct populations, there is the additional possibility of differences due to the cumulative effects of genetic sampling, or random drift, and the influence of natural selection, which may have favoured different gene arrays in different populations.

Qualitatively different diets will alter the relative contribution of different heterozygous loci to the total genetic variability. To take an example from a species a little closer to man, Falconer (1960) has shown that selection in the mouse for body-weight at the age of 3 weeks, on either a high or a low plane of nutrition, leads regularly to differences in carcass composition, such that, when fed on the highplane diet, mice selected on this diet have considerably higher fat content than those selected for ostensibly the same character on the low-plane diet. Also, the expression of the genetic differences between the strains differed according to diet. On the highplane both strains showed a phenotypic difference, but on the low-plane only the line selected on that diet showed the effects of selection. There are, of course, other strong indications that proneness to obesity may be considerably influenced by genotype (Davenport 1923; Steinberg, 1960; Fenton, 1960).

If genetic variation regularly contributes to individual variation in response to differences in nutrition, we should like to know relatively how important it is. In man, this poses a question which cannot be answered accurately, since we cannot apply the various genetic techniques we use in laboratory animals. In man, the study of polygenic variation for any trait which can be influenced by the environment is very difficult. For traits such as stature or say serum cholesterol level (Schaefer, Adlersberg \& Steinberg, 1958), a direct approach has been based on the correlation between relatives, especially between parents and offspring and between sibs, making due allowance for possible correlation between parents and differences due to age, sex and any other relevant variable. But this method is likely to be of little use with 
respect to dietary response of one sort or another, where past history so often influences present need.

Another approach would be to compare the degree of resemblance in performance between members of identical and non-identical twin pairs and also pairs of ordinary sibs. For various other criteria this procedure has provided a rough guide to the relative importance of genetic and environmental causes of variability. It is possible that a fortunate combination of circumstances might turn up identical and non-identical twin pairs who were willing to submit to control diets. Gradual accumulation of data of this sort would certainly be useful, provided there was sufficient standardization of methods to justify combining data of different workers.

For any such studies on man it would seem best to examine first the reactions known to show a high level of individual variation and, among these, there are few more suggestive examples than the ability to remain in calcium balance on a low calcium intake. There appears to be wide variation of opinion as to the origin of these striking individual differences. As a recent example we can note the careful work of Malm ( 1959) on a group of Norwegian prisoners whose calcium intake and excretion was studied over long periods. Particularly relevant here was their reaction to reduction in the mean daily intake of calcium from 967 to $459 \mathrm{mg}$. The reaction of the twenty-six subjects could be classified into the following categories.

(A) Immediate adaptation, always in positive balance or in balance.

(B) Initial negative balance, followed by better adaptation. This category could be subdivided into three groups:

(i) Compensatory adaptation with restoration of initial calcium loss.

(ii) New equilibrium at slight negative balance or balance, without restoring the initial calcium loss.

(iii) Marked negative balance followed by less marked, but still negative, balance.

(C) Continuous negative balance, no evidence of better adaptation.

Table 3 shows the distribution of these differing responses and poses the intriguing question of their origin, genetic, environmental, or both.

Table 3. Individual differences in response by Norwegian prisoners to a low calcium intake (after Malm, 1959)

$\begin{array}{ccc}\begin{array}{c}\text { Type of } \\ \text { response* }\end{array} & \begin{array}{c}\text { No. } \\ \text { of subjects }\end{array} & \begin{array}{c}\text { Mean no. of } \\ \text { days to adapt }\end{array} \\ \mathrm{A} & 3 & 0 \\ \mathrm{~B}_{1} & 7 & 68 \\ \mathrm{~B}_{2} & \mathrm{I} 2 & 95 \\ \mathrm{~B}_{3} & \mathrm{I} & \text { I40 } \\ \mathrm{C} & 3 & \text { No adaptation } \\ & \text { *See above. } & \end{array}$

Finally, the most practical approach to the general problem of genetic variation in response to diet must be indirect. There is great scope for systematic study of genetic variation of the nutritional requirements and growth reactions in the rat, to 
determine a kind of hierarchy of responses and relations, graded according to their relative resistance to environmental influence. 'This knowledge could provide a rough yardstick for application to man and could at least indicate responses in which genetic determination will be pre-eminent. Although there is need for caution in extrapolating from the behaviour of inbred lines, which may be regarded as rather aberrant genotypes, comparison of inbred lines and crosses could clarify the order of heterogeneity to be expected. There is also a real need of selection for differing responses on controlled diets, not only to study genetic variation but, perhaps more important, to follow the correlated changes in physiology and development. This method is potentially a very powerful one for investigating metabolic interrelationships, and in determining how far genetic differences in reaction to particular combinations of nutrients involve either minor differences in metabolic pathways or more regular patterns of change. There is a whole field of inquiry just waiting to be developed along these lines.

Very relevant here are the studies of Ross (1959) in relation to the effects on the growth of rats of diets differing in the levels of protein, carbohydrate and calories, but otherwise adequate. He has followed differences in life expectancy, the incidence of pathological changes, and has also measured some twenty-three hepatic enzymes associated with energy and oxidation, phosphate, carbohydrate and protein metabolism. He found characteristic changes in enzyme pattern with age and showed that protein deprivation could lead to enzyme patterns characteristic of younger individuals. Comparison of the levels of enzyme activity showed individual variation in each parameter and an increase in variance with age. But there was also striking evidence of dynamic equilibrium within individuals, so that when there was a low level of activity for one enzyme there were corresponding increases in the activity of others within that individual. There is thus greater biochemical stability between individuals than might appear from records of assays on only part or a few parts of the system. It would be valuable to know the effects of genetic differences on enzyme patterns of this kind. Comparison of inbred lines and crosses would be informative here, in view of the widely held view that the degree of dynamic adjustment possible in an inbred line will, on average, be lower than that in a cross.

Finally we may ask whether or not widely separated human populations differ in minimal requirements, or in the nature of their reaction to low levels of particular nutrients. If people have been exposed to malnutrition for many generations, severe enough to affect survival and reproduction, it is reasonable to suppose that genetic differences in tolerance will enter the picture: natural selection will have occurred. The effectiveness of such selection in altering gene frequencies will be lowered by fluctuations in the biochemical causes of malnutrition, but in populations confined to a few staple foods, by necessity, custom or taboo or by all three, there would appear to be ample scope for adaptation to such diets. A high level of infectious disease might accentuate the effects of such adverse diets in synergistic fashion. Although there is not much evidence that malnutrition affects susceptibility to many diseases, it would be as well to recall the observations of Schneider (195I) who showed that deficient diets led to greater mortality when rats of intermediate 
resistance were exposed to Salmonella strains of intermediate virulence. So, a high incidence of infection, coupled with an inadequate and insufficiently varied diet, could combine to promote genetic variation between populations. There are the familiar differences between peoples in stature and in ratio of bone width to length, and these have been correlated with protein and calcium intake. Evidence that people such as the Japanese grow to larger size when they emigrate to countries, such as the United States, where people eat more is sometimes quoted in favour of purely environmental origin of such differences (Greulich, 1958; Ohmori, 1958; György, I960). Environment may indeed play a large part in the final determination, but nutritional differences of the magnitude suggested by these comparisons may have exposed different gene arrays and thereby have allowed natural selection not only to establish differences in requirement for certain nutrients but also differences in the nature of the reaction to different limiting diets. We have to think in terms not merely of either genetic or environmental differences but of gene-environment interaction as well.

Admittedly, in the light of present knowledge or lack of it, such considerations are speculative, but greater awareness and better evaluation of genetic variation in relation to diet are likely to prove germane to the origin and maintenance of human diversity and the natural history of man.

I should like to thank Dr Reginald Passmore for drawing my attention to some useful recent papers.

\section{REFERENCES}

Davenport, C. B. (1923). Publ. Carneg. Instn, no. 329.

Falconer, D. S. (1 960). Genet. Res. 1, 91.

Fenton, P. H. (1 960). Amer. F. clin. Nutr. 8, 748.

Greulich, W. W. (1958). Science, 127, 515.

György, P. (1 g60). Amer. F. clin. Nutr. 8, 433.

Malm, O. J. (1959). Scand. F. clin. Lab. Invest. ro, Suppl. 36.

Ohmori, K. (1958). Keijo F. Med. 7, 157.

Prabhu, S. S. \& Robertson, F. W. (196I). Genet. Res. 2, 424.

Robertson, F. W. (1960a). Genet. Res. 1, 288.

Robertson, F. W. (1 960b). Genet. Res, 1, 305.

Robertson, F. W. (1960c). Genet. Res. r, 333 .

Ross, M. H. (1959). Fed. Proc. 18, I 190.

Schaefer, L. E., Adlersberg, P. \& Steinberg, A. G. (1958). Circulation, 17, 537.

Schneider, H. A. (I95I). Nutrition Sympositum Series no. 3. New York: The National Vitamin Foundation Inc. N.Y.

Steinberg, A. G. (1960). Amer. F. clin. Nutr. 8, 752. 